\title{
Optimization of Balanites aegyptiaca seeds supply chain for biofuel production in West Africa sahelian regions
}

\author{
Marie Sawadogo; Linda D. F. Bambara \\ Laboratoire Biomasse Energie et Biocarburants (LBEB) \\ $2 \mathrm{iE}$ \\ Ouagadougou Burkina Faso
}

\author{
Joël Blin \\ UPR Biomasse Energie \\ CIRAD \\ Montpellier France
}

\author{
Didier Anciaux ; Daniel Roy \\ Laboratoire LGIPM \\ Université de Lorraine \\ Metz France
}

\begin{abstract}
Balanites aegyptiaca (B. aegyptiaca) is one of the plant species targeted and already studied for the production of oilseed-based biofuels. This paper presents an optimization model of B. aegyptiaca seeds supply chain in West African sahelian context. It has been developed considering the West Africa rural context where family farming is mainly practiced. The model has been applied to a theoretical case of study in the sub sahelian region of the Burkina Faso. Four scenarios on the location of the pre-processing and on the transportation means used between $B$. aegyptiaca feedstock growing areas and feedstock gathering points (GP) have been performed. Considering different feedstock growing areas with different territorial yield, and different transportation means, the results show that the most efficient option is the cart for transportation between the feedstock growing areas and the GP with the location of the pre-processing at the GP. The results also show that more than $65 \%$ of the seeds cost price at the biorefinery gate is composed of the transportation costs.
\end{abstract}

Keywords- biomass conversion; Balanites aegyptiaca; optimization; supply chain

\section{INTRODUCTION}

Balanites aegyptiaca (B. aegyptiaca) is one of the plant species targeted and already studied for the production of oilseed-based biofuels [1]-[5]. The production of biofuels from this plant is particularly of interest for West African sahelian regions. In these sahelian regions, $B$. aegyptiaca is one of the dominant plant species [6] and the trees grows in the wild without irrigation nor fertilizer and maintenance. This is an advantage for biofuel production because no investment is needed for oilseeds growing. However, because they grow in the wild, the plants like B. aegyptiaca have a wide and scattered geographical distribution over the territory where they grow. This makes difficult and expensive the logistics operations such as collection and transport of the feedstock; the logistics costs can represent up to $35 \%$ of the total production cost of biofuels according to [7].
For a profitable and competitive exploitation of the $\mathrm{B}$. aegyptiaca for biofuel production, it is therefore important to pay a particular attention to the seeds supply chain [8]. This ensures that the biorefinery will be supplied with seeds in the required quality, quantity and an acceptable cost.

The work presented in this paper aims to determine the optimal conditions of B. aegyptiaca seeds supply to a biorefinery in west African sahelian regions. These optimal conditions are determined through a mathematical model that allows at considering the harvesting and the gathering of the seeds in the wild and their transport, and pre-processing.

\section{METHODOLOGY}

The model proposed in this paper is a mixed integer linear program (MILP) for strategic decisions related to the configuration of the B. aegyptiaca seeds supply chain for biofuel production in West Africa sahelian areas. These decisions concern the location and the allocation of the feedstock growing areas and of the feedstock gathering points (GP). They also concern the location of the preprocessing operations and the amounts of feedstock to transport.

\section{A. The problem and assumptions}

Let us consider a biorefinery located in a region of interest and having an annual demand for Balanites seeds or fruits. Depending on the choice of the pre-processing (depulping, dehulling, drying ...) location, the demand for feedstock of the biorefinery can represent the non-pretreated raw feedstock or the pretreated feedstock. In the region of interest, several feedstock growing areas have been identified in the wild around the biorefinery. Feedstock growing areas are sites where B. aegyptiaca trees are well-represented. To facilitate the delivery of the feedstock to the biorefinery (mainly the mobilization of labor for loading, unloading and weighing of feedstock), all the feedstock harvested must pass through a Gathering Point (GP). The GP indeed allow bringing together 
large amounts of feedstock. There are 2 possible locations for the pre-processing: "at the GP" or "at the biorefinery". Due to geographical constraints, several GP sites are selected in advance. The transports of feedstock between the GP are not possible. The biorefinery must be supplied with the demand for feedstock so as to minimize the total supply costs and satisfy some constraints.

\section{B. Mathematical formulation}

The mathematical formulation of the problem consists of an objective function to minimize (1) and a set of constraints (5) to (21). The objective function is composed of the feedstock harvesting costs (2), feedstock transportation costs from the feedstock growing areas to the GP (3), and feedstock transportation costs from the GP to the biorefinery (4). The elements that compose the mathematical model are stated and described in the section 1) and 2).

\section{1) Sets and parameters description}

Sets: feedstock growing areas of the B. aegyptiaca trees are represented by the index $i$, GP by the index $j$, biorefinery by the index $k$, transportation means by the index $m$, and preprocessing location sites by the index $p$.

Parameters: $Q_{k}$ is the annual demand for non-pretreated raw feedstock. This demand include feedstock loses that may occur during the supply. $\alpha$ is the remaining fraction of feedstock after the pre-processing. $\tau$ is a tortuosity factor. $S T_{i}$ is the total surface area of a feedstock growing area. Rend $_{i}$ is the territorial seed yield of a feedstock growing area. The territorial seed yield is the ratio of the total potential seed production on the feedstock growing area and of the surface area of this feedstock growing area. $a_{i, j}$ define the potential allocation of a feedstock growing area $i$ to a GP $j$. $C h_{i}$ is the unitary costs of harvesting in a feedstock growing area. $d_{i, j}$ is the distance between a feedstock growing area $i$ and a GP $j . d_{j, k}$ is the distance between a GP and the biorefinery. $x_{i, m}$ is a binary parameter that define if transportation mean $m$ is available in a GP $i . Q_{k, p}$ is the quantity of feedstock expected at the biorefinery depending on the location of the pre-processing. $Q_{\min _{j}}$ is the minimum quantity of feedstock required in a GP. $Q \max _{j}$ is the maximum capacity of a GP. $C_{m}$ is the unitary transportation cost of the mean of transportation $m$ and $C_{\text {truck }}$ is the unitary transportation cost of the truck. $P r_{m}$ and $P_{\text {truck }}$ are the fixed costs of the mean of transportation $m$ and of the truck. $\vartheta$ is the fruits picking rate. $W$ is the daily pay for harvesting workers. $\mathrm{Hr}$ is the prescribed number of work hours per day. $N T_{i}$ is the number of $\mathrm{B}$. aegyptiaca trees on each feedstock growing area. Prod is the annual amount of fruits produced by a B. aegyptiaca tree.

Variables: $S_{i}$ is the surface area of a feedstock growing area $i$. $y_{i}$ is a binary variable that decide the choice of a feedstock growing area $i . y_{j}$ is a binary variable that decide the choice of a GP $j . y_{p}$ is a binary variable that decide the location of the preprocessing. $Q_{i, j}$ is the quantity of feedstock to be transported from a feedstock growing area $i$ to a GP $j . Q_{j, k, p}$ is the quantity of feedstock to be transported from a GP $j$ to the biorefinery $k$ if the location of the pre-processing $p$ is chosen.

$$
\begin{aligned}
& \text { 2) Equations } \\
& \operatorname{Min}(H \operatorname{Cos} t+T \operatorname{Cos} t 1+T \operatorname{Cos} t 2)
\end{aligned}
$$

$$
\begin{aligned}
& S_{i} \geq 0 \forall i \\
& Q_{i, j} \geq 0 \forall i, j \\
& Q_{j, k, p} \geq 0 \forall j, k, p \\
& y_{i}, y_{j}, y_{p} \in\{0,1\} \forall i, j, p \\
& \text { HCost }=\sum_{i} \mathrm{Ch}_{i} . S_{i} \\
& \text { TCost } 1=\sum_{i, j, p, m} Q_{i, j, p} . d_{i, j} \cdot \tau . C_{m} . x_{i, m}+\sum_{m} P r_{m} . x_{i, m} \cdot y_{i} \\
& \text { TCost } 2=\sum_{j, k, p, m} Q_{j, k, p} \cdot d_{j, k} \cdot \tau . C_{\text {truck }}+P r_{\text {truck }} \cdot y_{j} \\
& S_{i} \geq y_{i} \forall i \\
& S_{i} \leq S T_{i} \forall i \\
& \sum_{i} y_{i} \geq 1 \\
& Q_{k, p}=Q_{k} \rightarrow p=\text { "at the biorefinery" } \forall p, k \\
& Q_{k, p}=Q_{k} . \alpha \rightarrow p \neq \text { “at the biorefinery” } \forall p, k \\
& \sum_{i, j} Q_{i, j} \geq \sum_{k} Q_{k} \\
& \sum_{j} Q_{i, j} \leq S_{i} \operatorname{Rend}_{i} \forall i \\
& Q_{i, j} \leq S_{i} \operatorname{Rend}_{i} \cdot y_{j} \forall i, j \\
& \sum_{j} Q_{i, j} \cdot a_{i, j} \leq y_{i} \forall i \\
& \sum_{i} Q_{i, j} \leq \operatorname{Qmax}_{j} \cdot y_{j} \forall j \\
& \sum_{i} Q_{i, j} \geq \operatorname{Qmin}_{j} \cdot y_{j} \forall j \\
& Q_{j, k, p}=\sum_{i} Q_{i, j} . \alpha \rightarrow p \neq \text { “at the biorefinery" } \forall j, k, p \\
& Q_{j, k, p}=\sum_{i} Q_{i, j} \rightarrow p=\text { “at the biorefinery" } \forall j, k, p \\
& \sum_{j, k} Q_{j, k, p} \geq \sum_{k} Q_{k, p} \cdot y_{p} \forall p \\
& \sum_{j, k, p} Q_{j, k, p} \geq \sum_{k, p} Q_{k, p} \cdot y_{p} \\
& \sum_{j} y_{j} \geq 1 \text { (20) } \\
& \sum_{p} y_{p}=1(21) \\
& C h_{i}=\left(\operatorname{Rend}_{i} . W\right) /(\vartheta . H r) \forall i \\
& \text { Rend }_{i}=\operatorname{Prod} . N T_{i} / S_{\mathrm{i}}
\end{aligned}
$$

Equation (5) and (6) ensure that the surface area mobilized by each chosen feedstock growing area will not exceed the available surface area of each feedstock growing area. Equation (7) states that several feedstock growing areas can be chosen. Equations (8) and (9) calculate the quantity of feedstock required at the biorefinery depending on the location of the pre-processing. Equation (10) ensures that the sum of the quantities of feedstock harvested and transported between the chosen feedstock growing area and the chosen GP have to satisfy the required quantity of feedstock expected at the biorefinery. Equations (11) to (13) ensure that the quantities of feedstock transported from the chosen feedstock growing areas to the chosen GP, must not exceed the quantities of feedstock that can be harvested on each feedstock growing area. Equation (14) ensures that the maximum capacity of a selected GP must not be exceeded. Equation (15) ensures that the minimum demand of each selected GP have to be satisfied. Equation (16) and (17) calculates the quantity of feedstock to be transported between each selected GP and the biorefinery 
depending on the choice of the pre-processing location. Equation (18) and (19) ensures that the quantities of feedstock transported between the chosen GP and the biorefinery, have to satisfy the demand of the biorefinery depending on the location of the pre-processing. Equation (20) ensures that several GP can be chosen. Equation (21) ensures that only one location of the pre-processing can be chosen. Equation (22) calculates the unitary costs of harvesting on a feedstock growing area. Equation (23) calculates the territorial seed yield of a feedstock growing area.

\section{CASE STUDY}

The model has been implemented on a theoretical case study of B. aegyptiaca seeds supply chain. The case study was constructed on the basis of common practices in Burkina Faso. Data and assumptions are retrieved from peer-reviewed literature and expert opinions. Furthermore, the costs calculated reflect the real costs that can be observed in Burkina Faso.

The considered biorefinery is in a region with 222 growing area of B. aegyptiaca trees and 35 potential GP.

The geographical coordinates and the surface of the feedstock growing areas, the GP and the biorefinery have been randomized once and the resulting supply network has been used for all the study. The surface area value of each feedstock growing area has been set to $10 \mathrm{ha}$. A productivity of $52 \mathrm{~kg}$ of fruits per tree was retained for all the feedstock growing areas [4]. A B. aegyptiaca trees density ranging from 25 trees/ha to 40 trees/ha was randomly distributed for each of the feedstock growing area. According to [9], a density ranging from 25 to 50 trees per ha is acceptable for a region where B. aegyptiaca is well-represented.

All distances are Euclidean distances to which a tortuosity factor has been applied. With no knowledge on the road network of the study area, the value of the tortuosity factor can only be estimated. For this study, its value has been set to $\sqrt{2}$ (we have considered that the straight-line distance is the diagonal of a square, the sides of which represent the actual path).

The minimum demand of each GP is about 10 tons which represent the load capacity of the trucks commonly used for the transportation of goods in Burkina Faso. The maximum capacity of each GP is unlimited.

The demand for B. aegyptiaca seeds of the biorefinery is 200 tons/year. Each fruit has a weight of about 5 to $8 \mathrm{~g}$. It consists of an epicarp (5 to $9 \%$ of its weight), an edible mesocarp or pulp ( 28 to $33 \%$ of its weight), an endocarp (49 to $54 \%$ of its weight). The endocarp surrounds a the seed ( 8 to $12 \%$ of the weight of the fruit) [10]-[12]. The seed contains between $46 \%$ and $55 \%$ of its weight in vegetable oil [13]

On this basis, the demand for non-pretreated feedstock of the biorefinery is 2000 tons/year if the kernel represents $10 \%$ of the fruit weight.

The harvesting costs per hectare are estimated based on the corresponding labor costs. A man-day is assumed to equate 8 hours. The minimum daily wage for farm workers is 1363.91 $\mathrm{XOF} /$ day in Burkina Faso (XOF is West African CFA francs).
The harvesting labor costs are calculated based on a fruits picking rate about $9.24 \mathrm{~kg} / \mathrm{h}$ found by [14] in the locality of Barsalogho in Burkina Faso.

Carts pulled by donkeys are considered for the transportation between the feedstock growing areas and the GP. Fifteen years old Trucks are considered for the transportation between the GP and the biorefinery. The unitary transportation cost by carts and tricycles are estimated on the basis on the minimum wage for the driver, the maximum load per travel, the velocity of each mean and the fuel consumption for the tricycle. For carts, this cost is about $136 \mathrm{XOF} /$ ton.km. Its maximum load is about 0.5 tons [15] and the maximum traveling distance covered in a day is $20 \mathrm{~km}$ when considering a velocity of $5 \mathrm{~km} / \mathrm{h} \mathrm{[15]} \mathrm{and} \mathrm{a} \mathrm{work} \mathrm{day} \mathrm{of} 8$ hours. For tricycles, this cost is $27 \mathrm{XOF} /$ ton. $\mathrm{km}$. Its maximum load is 1.1 ton and its fuel consumption is $3.51 / 100 \mathrm{~km}$ [14]. The maximum distance covered in a day is $160 \mathrm{~km}$ when considering a velocity of $40 \mathrm{~km} / \mathrm{h}$ and a work day of 8 hours. For truck, this cost is $52 \mathrm{XOF} /$ ton.km for roads with no bitumen [16]. Its maximum load is about 10 tons. The costs for each mean are presented in TABLE 1. Only fixed costs arising from the transport of the Balanites fruits or seeds have been calculated. Indeed, in West African rural areas where family farming is practiced, the goods means of transportation are not devoted to a single activity. In this case study, it was considered that the means of transportation are used only from October to December, i.e. 3 months per year for the biorefinery supply (it is the case in Barsalogho). This period of 3 months is in the ripening period of the Balanites fruits which goes from October to February according to [17].

Four scenarios are performed. In scenario 1, the carts pulled by donkeys are used for the feedstock transportation between the feedstock growing areas and the GP and two locations of the pre-processing (at the GP and at the biorefinery) are possible. In scenario 2 the tricycles are used for the feedstock transportation between the feedstock growing areas and the GP; for this scenario, two locations of the pre-processing (at the GP and at the biorefinery) are possible. In scenario 3 , the carts pulled by donkeys are used for the feedstock transportation between the feedstock growing areas and the GP and only one location of the pre-processing (at the biorefinery) is possible. In scenario 4 the tricycles are used for the feedstock transportation between the feedstock growing areas and the GP and only one location of the pre-processing (at the biorefinery) is possible.

TABLE I. ATTRIBUTES OF THE TRANSPORTATION MEANS

\begin{tabular}{|c|l|l|l|}
\cline { 2 - 4 } \multicolumn{1}{c|}{} & Tricycle & \multicolumn{1}{c|}{ Cart } & Truck \\
\hline Interest rate & 0,05 & 0,05 & 0,05 \\
\hline Life time (year) & 5 & 5 & 10 \\
\hline Price (XOF) & 1000000 & 130000 & 4000000 \\
\hline Fixed cost (XOF) & 57744 & 7507 & 129505 \\
\hline Unitary cost (XOF/ton.km) & 27 & 136 & 52 \\
\hline
\end{tabular}




\section{RESULTS AND DISCUSSION}

The model has been implemented and solved in the optimization software Xpress IVE 7.9. The experiments were performed on an Intel Core i7- 5500U CPU $2.4 \mathrm{GHz}$ with $8 \mathrm{~GB}$ RAM on a 64-bit platform. The solution was obtained in less than 30 seconds using the simplex and barrier algorithms available in the software solver. The model determines the number of feedstock growing area among a set of feedstock growing areas previously identified and the optimal surface area of this feedstock growing areas to be harvested. It also determines the optimal number of GP to be opened among a set of GP previously identified, the allocation of each chosen feedstock growing area to at least one GP, the optimal quantity of harvested feedstock to be transported between each chosen feedstock growing area and GP, the optimal amount of feedstock to be transported between each chosen GP and the biorefinery, and finally the optimal location of the preprocessing.

For each scenario, the costs results are presented in Fig.1. The harvesting costs represent the costs per $\mathrm{kg}$ of $\mathrm{B}$. aegyptiaca fruits. The transportation costs represent the costs per $\mathrm{kg}$ of B. aegyptiaca seeds.

The $\mathrm{B}$. aegyptiaca seeds cost price at the biorefinery gate is $59.8 \mathrm{XOF} / \mathrm{kg}$ for scenario $1,64.6 \mathrm{XOF} / \mathrm{kg}$ for scenario $2,85.3$ $\mathrm{XOF} / \mathrm{kg}$ for scenario 3 and $80.7 \mathrm{XOF} / \mathrm{kg}$ for scenario 4 . These results show that the seeds cost price at the biorefinery gate is higher than the purchasing price of the B. aegyptiaca dry fruits at the biorefinery gate in the locality of Barsologho in the sub sahelian region of the Burkina Faso. The dry fruits purchasing price in this locality is $40 \mathrm{XOF} / \mathrm{kg}$. This low purchasing price in Barsalogho means that the actual logistical costs have not been taken into account when setting the dry fruits purchase price. This is supported by the fact that this price of $40 \mathrm{XOF} / \mathrm{kg}$ is the price of the non-pretreated fruits at the biorefinery gate. Moreover, this price of $40 \mathrm{XOF} / \mathrm{kg}$ takes into account the storage cost which has not been considered in the model describe in this paper.

Moreover, the results of the implementation show that the pre-processing location "at the GP" is always chosen for the scenario 1 and scenario 2 . The seeds cost prices for the scenarios with the pre-processing located at the biorefinery are indeed the highest. This makes sense because there is only $10 \%$ of the feedstock to be transported when the preprocessing is located at the GP. For this reason, we will continue the rest of the analysis of the results with scenarios 1 and 2.

The results of the implementation also show that 109 feedstock growing areas are chosen and 6 GP are opened for the scenario with the tricycle (scenario 2). For the scenario with the cart (scenario 1), 118 feedstock growing areas are chosen and 12 GP are opened. This can be explained by the short travel distance per day of the cart $(20 \mathrm{~km})$ compared to the travel distance per day of the tricycle $(160 \mathrm{~km})$.

The results of the chosen feedstock growing areas and of the chosen GP can be seen in Fig. 2 and Fig. 3. The red arrows represent the transport from the feedstock growing areas to the
GP. The blue arrows represent the transport from the GP to the biorefinery. The green points represent the chosen feedstock growing areas and the black points represent the non-chosen feedstock growing areas. The blue boxes represent the GP.

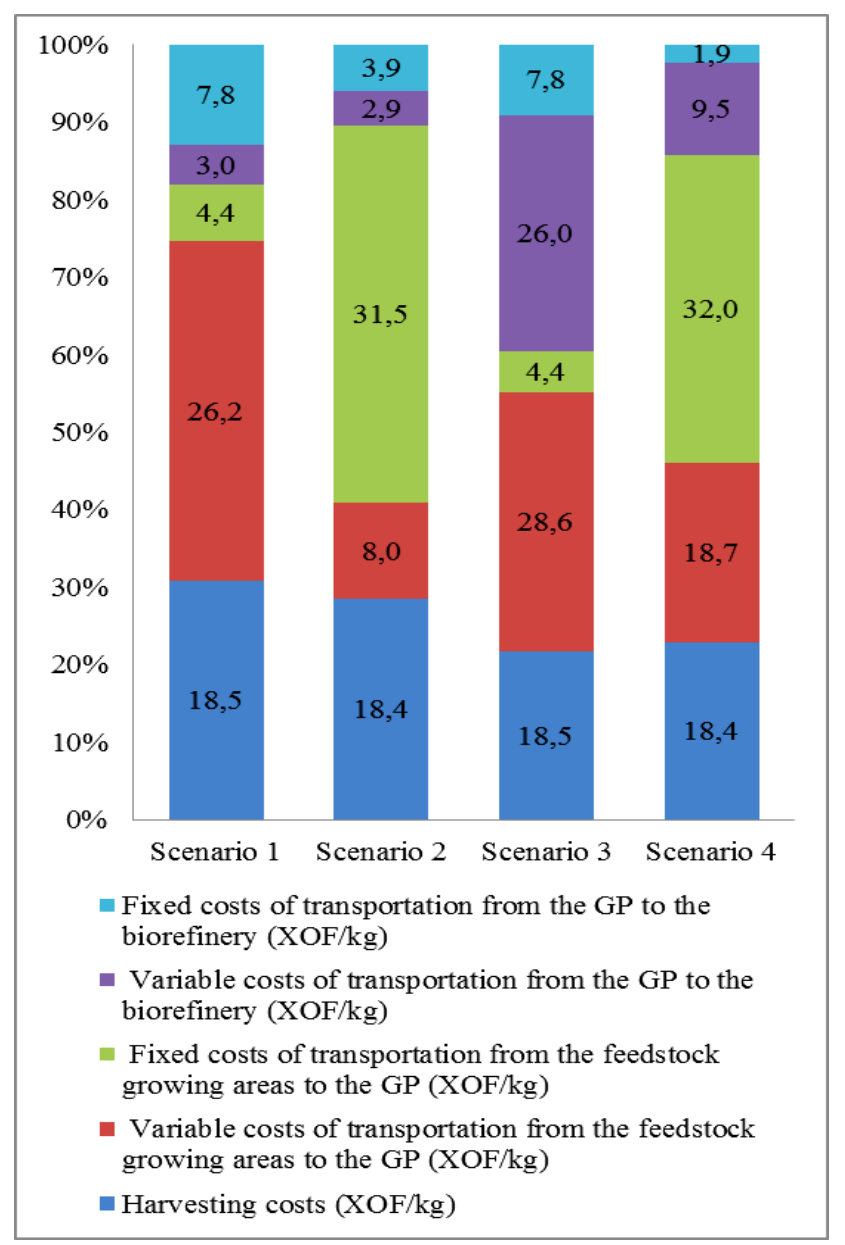

Fig. 1. Cost results

The harvesting costs are similar for the scenario 1 and 2 . They represent $31 \%$ of the seeds cost price at the biorefinery gate for scenario 1 and $29 \%$ of the seeds cost price at the biorefinery gate for scenario 2 . The transportation cost is 41.4 $\mathrm{XOF} / \mathrm{kg}$ for scenario 1 and is $46.2 \mathrm{XOF} / \mathrm{kg}$ for scenario 2 . This cost represents $69 \%$ of the seeds cost price at the biorefinery gate for scenario 1 and $71 \%$ of the seeds cost price at the biorefinery gate for scenario 2 . The harvesting and transportations costs results show that more than $65 \%$ of the seeds cost price at the biorefinery gate is composed of the transportation cost.

For scenario 1, the transportation cost between the feedstock growing areas and the GP is $30.6 \mathrm{XOF} / \mathrm{kg}$. It represents $74 \%$ of the total transportation cost. For the scenario 2 this cost of transportation between the feedstock growing area and the GP is $39.4 \mathrm{XOF} / \mathrm{kg}$. It represents $85 \%$ of the total transportation cost. This higher transportation cost for scenario 2 can be explained by the fixed costs of the tricycle that are 7.7 times higher than the fixed costs of the cart (TABLE 1.). 
It can also be seen from Fig.1. that the transportation cost is mainly affected by the purchasing cost of the transportation mean when the motor tricycles are used. When the carts pulled by donkeys are used, the total transportation cost is mainly affected by the variable costs of transportation.

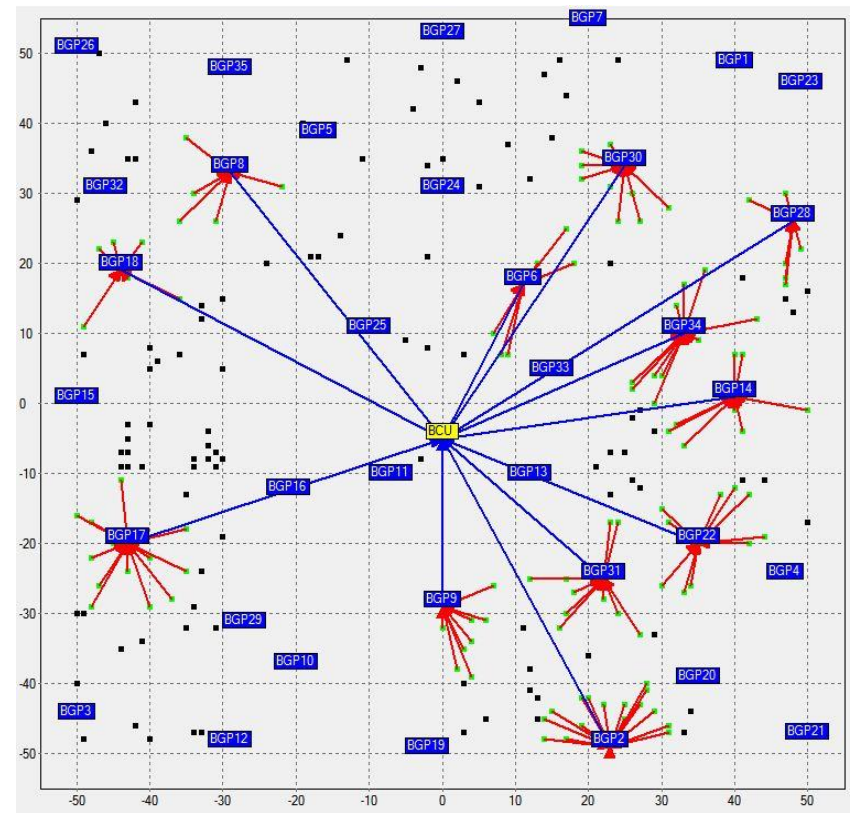

Fig. 2. The supply network in scenario 1

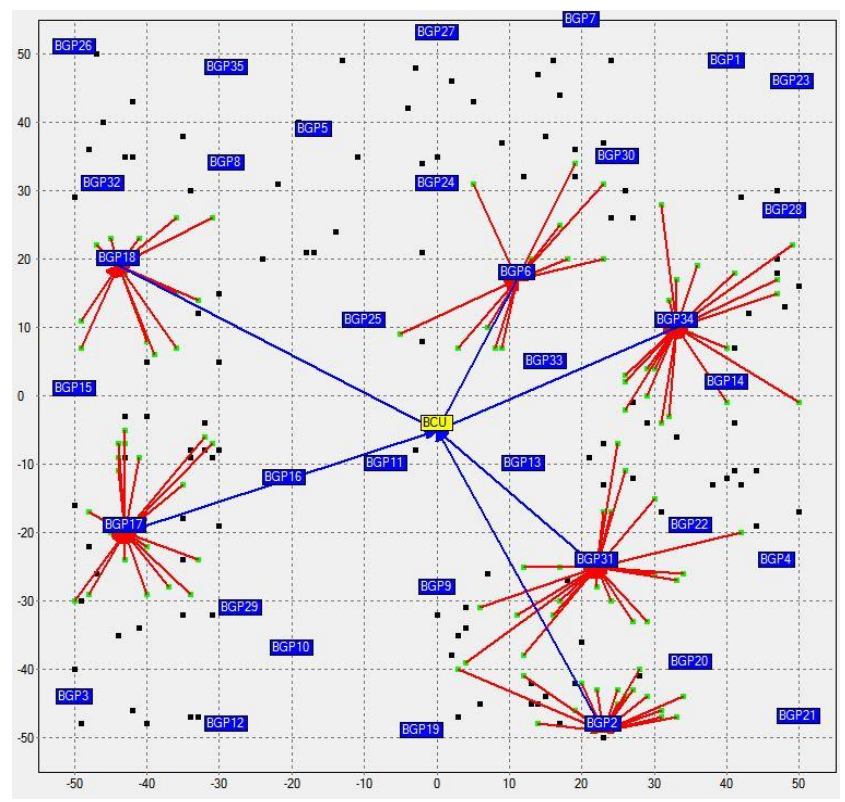

Fig. 3. The supply network in scenario 2

\section{CONCLUSION}

In this paper, a mathematical model for strategic decisions in Balanites aegyptiaca seeds supply chain for biofuel production in West African sahelian regions has been described. The functionalities and possibilities of the model developed have been demonstrated by its implementation on a theoretical case of study in the sub sahelian region of the
Burkina Faso. The results of the implementation show that more than $65 \%$ of the seeds cost price at the biorefinery gate is composed of the transportation cost.

Our future work will consist in a comparative study of the costs of B. aegyptiaca and Jatropha curcas seeds supply chain. The aim is to see if the cost price of the B. aegyptiaca seeds can be competitive compared to the jatropha's one.

\section{REFERENCES}

[1] J. C. J. Bart, N. Palmeri, and S. Cavallaro, "6 - Emerging new energy crops for biodiesel production," in Biodiesel Science and Technology, J. C. J. Bart, N. Palmeri, and S. Cavallaro, Eds. Woodhead Publishing, 2010, pp. 226-284.

[2] N. K. Patel and S. N. Shah, "11 - Biodiesel from Plant Oils," in Food, Energy, and Water, S. Ahuja, Ed. Boston: Elsevier, 2015, pp. 277-307.

[3] A. E. Atabani, A. S. Silitonga, I. A. Badruddin, T. M. I. Mahlia, H. H Masjuki, and S. Mekhilef, "A comprehensive review on biodiesel as an alternative energy resource and its characteristics," Renew. Sustain. Energy Rev., vol. 16, no. 4, pp. 2070-2093, 2012.

[4] B. P. Chapagain, Y. Yehoshua, and Z. Wiesman, "Desert date (Balanites aegyptiaca) as an arid lands sustainable bioresource for biodiesel," Bioresour. Technol., vol. 100, no. 3, pp. 1221-1226, 2009.

[5] S. J. Deshmukh and L. B. Bhuyar, "Transesterified Hingan (Balanites) oil as a fuel for compression ignition engines," Biomass Bioenergy, vol. 33, no. 1, pp. 108-112, Jan. 2009.

[6] J.-M. Boffa, Les parcs agroforestiers en Afrique subsaharienne. Food \& Agriculture Org., 2000.

[7] K.-Q. Fan, P.-F. Zhang, and Z. J. Pei, "An assessment model for collecting and transporting cellulosic biomass," Renew. Energy, vol. 50, pp. 786-794, 2013.

[8] L. G. Papageorgiou, "Supply chain optimisation for the process industries: Advances and opportunities," Comput. Chem. Eng., vol. 33, no. 12, pp. 1931-1938, 2009.

[9] J. B. Hall, "Ecology of a key African multipurpose tree species, Balanites aegyptiaca (Balanitaceae): the state-of-knowledge," For. Ecol. Manag., vol. 50, no. 1, pp. 1-30, 1992.

[10] B. Chapagain and Z. Wiesman, "Variation in diosgenin level in seed kernels among different provenances of Balanites aegyptiaca Del (Zygophyllaceae) and its correlation with oil content," Afr. J. Biotechnol., vol. 4, no. 11, 2005.

[11] C. A. Okia, J. Kwetegyeka, P. Okiror, J. M. Kimondo, Z. Teklehaimanot, and J. Obua, "Physico-chemical characteristics and fatty acid profile of desert date kernel oil.," Afr. Crop Sci. J., vol. 21, no. Suppl. 3, pp. 723-734, 2013.

[12] B. P. Chapagain, "Characterization of Desert Date (balanites Aegyptiaca) Saponins and Their Biological Activities," Ben-Gurion University of the Negev, Beer-Sheva, 2006.

[13] A. Varshney and M. Anis, Trees: Propagation and Conservation: Biotechnological Approaches for Propagation of a Multipurpose Tree, Balanites aegyptiaca Del. Springer Science \& Business Media, 2014.

[14] Y. Füllemann, "Evaluation of vegetable fuel oil production potential in rural Burkina Faso," EPFL, 2015 unpublished.

[15] P. Lhoste, M. Havard, and É. Vall, La traction animale. Quae, 2010.

[16] AMASSA AFRIQUE VERTE MALI, "Manuel d'information sur le transport des produits agricoles au Mali." May-2009 .

[17] M. Ndoye, I. Diallo, and Y. K. Gassama/Dia, "Reproductive biology in Balanites aegyptiaca (L.) Del., a semi-arid forest tree," Afr. J. Biotechnol., vol. 3, no. 1, pp. 40-46, Jun. 2004. 